\title{
Development of a rapid and sensitive analytical method for the determination of ${ }^{129}$ I by ICP-MS/MS
}

\author{
TAKESHI OHNO ${ }^{1}$, HINA SATO ${ }^{1}$ \\ ${ }^{1}$ Department of Chemistry, Faculty of Science, Gakushuin \\ University, Mejiro 1-5-1, Toshima-ku, Tokyo, Japan, \\ 171-8588, takeshi.ohno@gakushuin.ac.jp
}

The long-lived radioiodine isotope ${ }^{129} \mathrm{I}$ (half-life: $\left.1.57 \times 10^{7} \mathrm{y}\right)$ is one of the most important radionuclides released from nuclear fuel reprocessing plants and nuclear accidents into the environment. This nuclide has provided useful information on the behavior of radioiodine in the environment. In addition, ${ }^{129} \mathrm{I}$ has been used as a tool to reconstruct the distribution of ${ }^{131}$ I (half-life: 8 days) at nuclear accidents.

Advances in inductively coupled plasma tandem quadrupole mass spectrometry with a reaction cell (ICPMS/MS) have enabled us to determine ${ }^{129} \mathrm{I}$ in a number of environmental matrices. However, there are a few challenges in measuring iodine-129. The most serious problem is the spectrum interferences of ${ }^{127} \mathrm{IH}_{2}{ }^{+}$and other polyatomic ions, such as ${ }^{97} \mathrm{MoO}_{2}{ }^{+}$, etc. The aim of this study is the development of a rapid and sensitive analytical method applicable to the measurement of the radioiodine.

For reducing spectrum interferences, we developed a gas sample introduction system equipped with three channels used for mixing $\mathrm{HNO}_{3}, \mathrm{NaNO}_{2}$, and sample solutions. The iodine in the solution was oxidized and vaporized by $\mathrm{HNO}_{3}$ and $\mathrm{NaNO}_{2}$. The sample preparation procedure used in this study consists of the separation of iodine from soil samples by pyrohydrolysis.

The ${ }^{127}$ I sensitivity of the developed method improved more than 10 times higher than that of the conventional solution nebulizing introduction. We think the improvement of transduction efficiency and ionization efficiency can be achieved. When iodine is separated from the soil sample by thermal hydrolysis, molybdenum is also volatilized and trapped together. In this study, by vaporizing iodine and introducing the gaseous iodine into ICP-MS/MS, the interference of polyatomic ${ }^{97} \mathrm{MoO}_{2}{ }^{+}$could be reduced compared to the solution introduction. While AMS requires complex pretreatments such as solvent extraction, this analytical method does not need the time-consuming pretreatments. 